This article was published in Journal of Materials Chemistry A, 2 (7), 2028-2032, 2014 http://dx.doi.org/10.1039/c3ta14593j

\title{
Transparent graphene-based counter-electrodes for iodide/triiodide mediated dye-sensitized solar cells**
}

\author{
Rui Cruz ${ }^{a}$, João Pedro Araújo ${ }^{\text {b }}$, Luísa Andrade a, Adélio Mendes*a
}

Broader Context Dye-sensitized solar cells (DSCs) have been regarded as a promising cheaper alternative to conventional photovoltaic systems. Fostering large-scale applications, many efforts have been made to replace platinum as the catalytic material of the electrocatalytic reaction that takes place at the counter-electrode (CE) side (cathode) of the DSC due to its scarce nature and high price. To date, there was no other material capable of matching Pt electrocatalytic activity for the $13^{-} / \mathrm{I}^{-}$redox couple (the most used electrolyte system), associated with a high optical transparency of the electrode. Although transparency is not mandatory for a DSC, it is highly appreciated as it increases the product value by enabling its use in building integrated applications (BIPV), as well as in other solutions such as tandem cells. Consequently, an important challenge emerges in order to find a proper substitute for Pt as the CE material of a DSC. Here we report the development of a novel CE capable of delivering simultaneously high efficiency and transparency for iodine-based electrolyte systems in DSCs. The presented CE is based on a structured film of oxidized graphene nanoplatelets applied over metal (nickel) nanoparticles, with a very simple manufacturing process. Both materials and processes should enable the fabrication of a cheaper DSC.

A new highly transparent and low cost counter-electrode for dye-sensitized solar cells was fabricated, comprised of a structured graphene film over nickel nanoparticles. Annealed nickel particles induced an enhanced restoration of graphene double bonds, which led to cells with energy conversion efficiencies similar to those using a conventional platinum electrode.

Dye-sensitized solar cells (DSCs) are a promising new alternative photovoltaic technology due to the lower manufacturing costs and potential for high-energy conversion ${ }^{1,2}$. Typically a DSC is comprised of a porous nanocrystalline titanium oxide film (TiO2) decorated with a sensitizer and a platinum counter-electrode (CE), both deposited on top of a transparent conductive oxide glass, and an liquid electrolyte containing iodide/triiodide $\left(13^{-} / \mathrm{I}^{-}\right)$redox couple.

Throughout the years attempts have been made to replace Pt as the catalyst material in DSCs with potentially cheaper carbonaceous materials, either in their pristine or composite forms. ${ }^{3,4}$ However, as the performance of these materials is strongly affected by the available surface area for reaction, ${ }^{4,5}$ it is necessary a large amount of carbon for efficient catalysis making the electrodes opaque and bulky, ${ }^{4}$ and thus undermining the DSC's transparency properties offered by the Pt CE. Graphene has been gathered interest in recent years as a potential candidate for the replacement of traditional Pt as a CE material in a DSC. Graphene, an atomically thick sheet composed of $\mathrm{sp}^{2}$ carbon atoms arranged in a flat honeycomb structure, ${ }^{6}$ is a material with very high specific surface area, high optical transmittance and good electrocatalytic properties. ${ }^{7-9}$ Moreover, it is a relatively cheap, abundant and non-toxic material ${ }^{10,11}$ and resistant to 
electrocorrosion. ${ }^{8}$ The electroactivity of graphene is induced by lattice defects and attached oxygen-functional groups ${ }^{8,12}$ but a sufficient high electrical conductivity should also be maintained. ${ }^{6}$ Only just now have graphene-based CEs been proved to match and even surpass Pt as the premier catalytic material for DSCs but in different redox couple systems (ferrocene ${ }^{13}$ or cobalt ${ }^{14,15}$ ). Nevertheless such results have not been obtained yet for the $13^{-} / \mathrm{I}^{-}$redox couple electrolyte.

Recently we have shown that the thermal reduction of exfoliated graphene oxide platelets could yield promisingly efficient CEs for the $13^{-} / \mathrm{I}^{-}$redox reaction, while having high transparency (transmittance higher than $80 \%$ at $550 \mathrm{~nm}$ ). ${ }^{16}$ The presented procedure enabled averting the need to pre-reduce oxidized and defected graphene nanoplatelets prior to film deposition, thus reducing the manufacturing complexity. Nevertheless, there was a shortcoming of efficiency due to high charge-transfer resistances mainly caused by poor electronic connection between graphene platelets and the substrate. The present contribution unveils an innovative CE that uses oxidized graphene nanoplatelets (mGOM5) spray-deposited on top of a covered-glass fluorinedoped tin oxide (FTO) substrate previously decorated with nickel nanoparticles (mGOM5/Ni CE), followed by thermal annealing under an inert atmosphere. The oxygen moieties and defects were introduced in a commercial graphene sample using the Hummers method. ${ }^{17}$

Atomic force microscopy (AFM) and scanning electron microscopy (SEM) images (Fig. 1) depict films of oxidized graphene nanoplatelets (mGOM5) that were deposited on top of the FTO surface before and after being thermally annealed. Before annealing the mGOM5 film appears to be only resting on top of the FTO surface; after annealing the film seems to sink into the typical FTO's topography ("mountain-crests" shapes), molding its structure to that landscape (Fig. 1c), and adhering to the FTO's surface. Such perception was validated by surface roughness measurements; the roughness of the surface was measured to be Ra $13.5 \mathrm{~nm}$ for the FTO surface, Ra $~ 9.7 \mathrm{~nm}$ for the nonannealed mGOM5 film and $\mathrm{Ra} \sim 12.5 \mathrm{~nm}$ for the annealed one.

Structured mGOM5/Ni films as described in the Experimental Section, were also prepared. Once again, and even in the presence of the nickel nanoparticles, annealing caused the molding of the graphene platelets onto the FTO's surface. It should be mentioned that the depositions of all mGOM5-based films were prepared to display transmittance values above $80 \%$ at a wavelength of $550 \mathrm{~nm}$ ( $\tau 550 \mathrm{~nm})$. It can be seen that the mGOM5 film created by spray deposition did not covered completely the FTO/Ni substrate (Fig. $1 \mathrm{~d}$ and 1 e), leaving some Ni particles exposed. Although some $\mathrm{Ni}$ particles are exposed to the electrolyte, neither corrosion nor leaching of Ni occurred. ${ }^{18,}$ 19 Energy Dispersive Spectroscopy (EDS) scanning performed on the darker areas signaled the presence of carbon; the graphene layer is so transparent that the nickel particles underneath are clearly perceived (Fig. 1e.) 

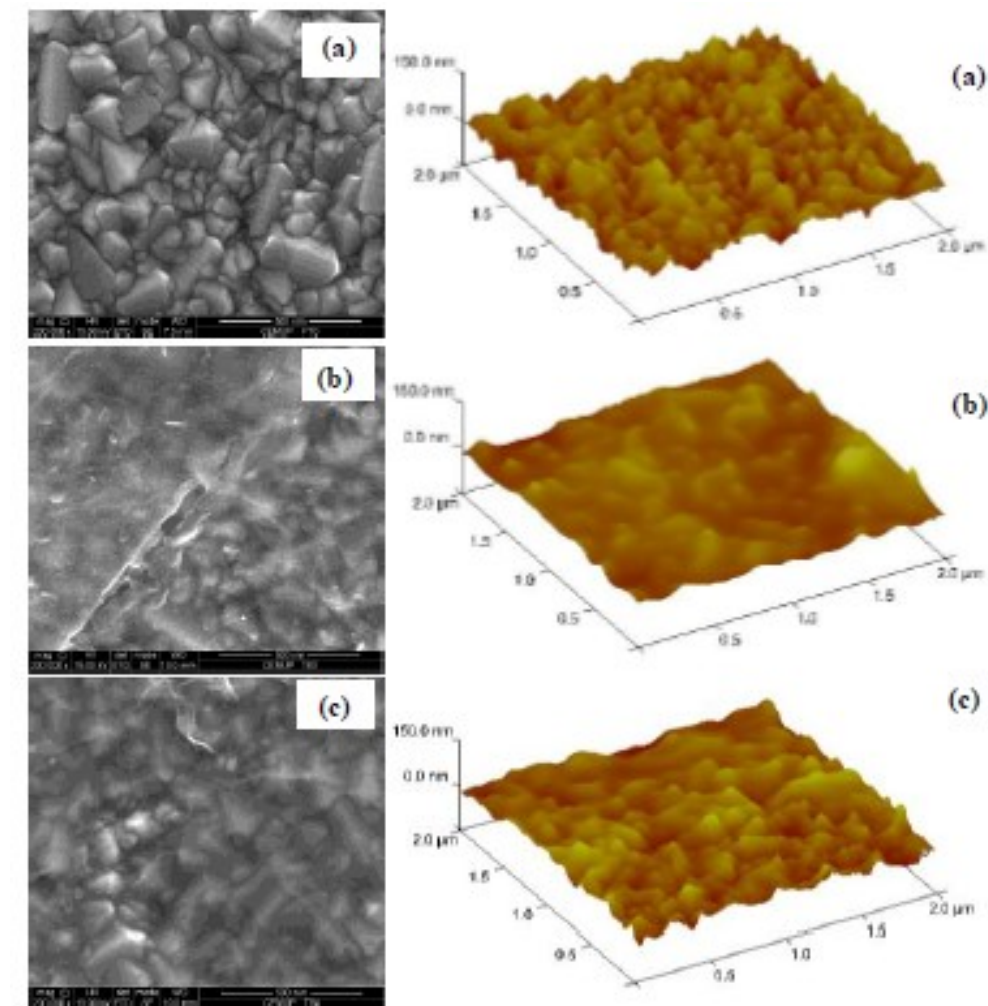

(b)
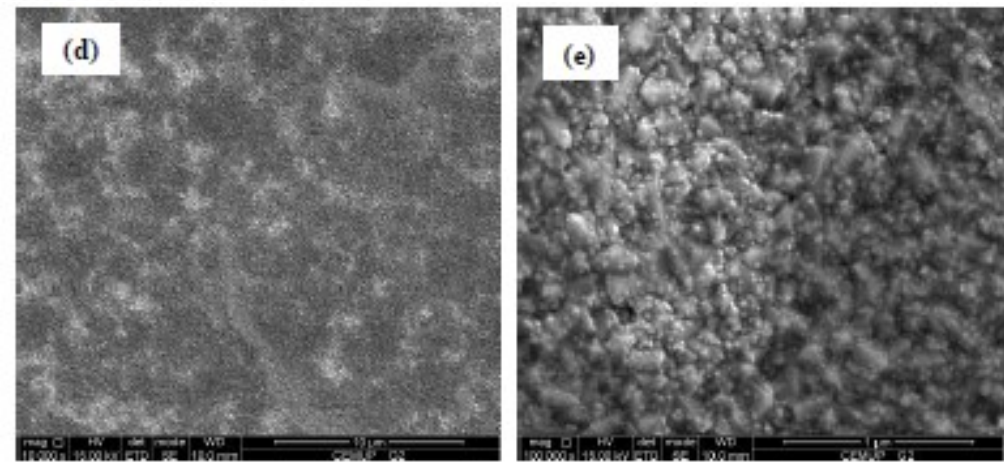

Fig.1 AFM and SEM images of (a) the pristine FTO surface, (b) film of oxidized graphene nanoplatelets (mGOM5) deposited on FTO surface prior to thermal annealing and c) after annealing - notice the moulding effect of the graphene platelets onto the FTO's surface; (d) and (e) SEM images of mGOM5/Ni Ce sample after thermal annealing. Concerning Fig. 1e it should be highlighted the transparency of the graphene layer that allows seeing nickel particles underneath them. Each mGOM5 nanoplatelet is $\sim 8 \mathrm{~nm}$ thick and has a length of ca. $0.6 \mu \mathrm{m}$ to $2 \mu \mathrm{m}$ (Supplementary Information section 1 ).

Fig. 2 and Table 1 show the current-voltage characteristics obtained for DSCs assembled with an mGOM5/Ni CE and with the reference Pt CE. Two different solvents (water and ethanol) used to disperse the mGOM5 platelets were tested. The DSCs fabricated with the $\mathrm{mGOM} 5 / \mathrm{Ni}$ CEs that were prepared with the two solvents yielded similar efficiencies ( $\eta=7.51 \%$ in water vs. $\eta=7.51 \%$ in ethanol), hinting that the type of solvent used does not affect the CE's performance. The mGOM5/Ni CEs prepared, using water or ethanol, yielded DSCs with efficiencies very similar to the DSC with the platinum CE - 7.45\%. The mGOM5/Ni CEs yielded a higher fill factor (FF) than the Pt CE (0.640 vs. 0.605$)$. The 
mGOM5/Ni CE prepared with ethanol was more transparent than the one prepared with water ( $\tau 550 \mathrm{~nm}=91.8 \%$ vs $80.6 \%$ ) and almost as transparent as the Pt CE ( $\tau 550 \mathrm{~nm}=$ $92.0 \%)$.

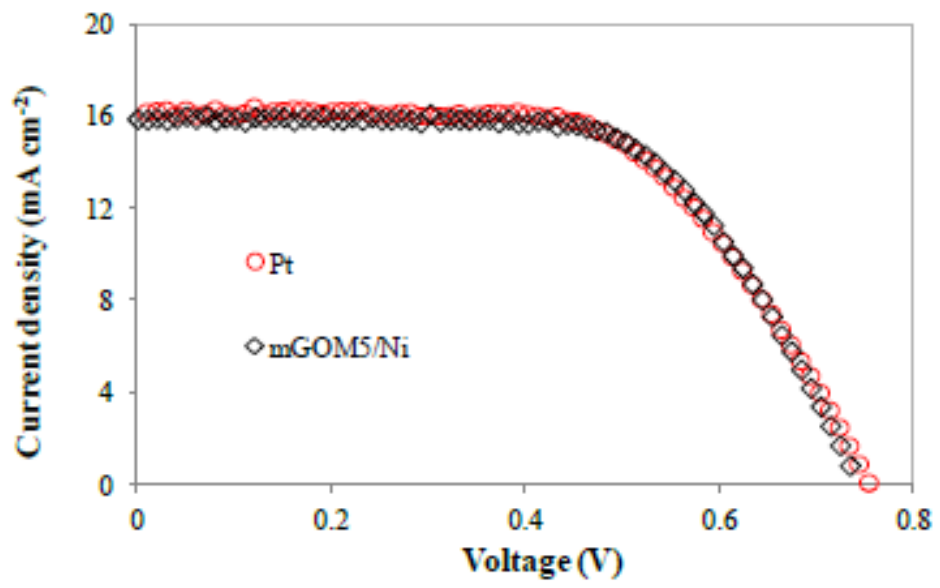

Fig 2. I-V characteristics of DSCs assembled with $\mathrm{mGOM} 5 / \mathrm{Ni}$ (in water) and with Pt CEs. Measurements carried out at 1 sun $\left(100 \mathrm{~mW} \bullet \mathrm{m}^{-2}, \mathrm{AM} 1.5 \mathrm{G}\right.$, room temperature). The mGOM5/Ni CEs fabricated using ethanol were not depicted due to overlap with mGOM5/Ni CEs fabricated using water.

Table 1. I-V parameters of DSCs assembled with the $\mathrm{mGOM} 5 / \mathrm{Ni}$ and $\mathrm{Pt} \mathrm{CE}^{[\mathrm{a}]}$, measured under one sun simulated sunlight $\left(100 \mathrm{~mW} \bullet \mathrm{m}^{-2}, \mathrm{AM} 1.5 \mathrm{G}\right.$, room temperature). Between parenthesis it is mentioned the solvent used for dispersing the mGOM5 platelets.

\begin{tabular}{cccccc}
\hline Counter-electrode & $V_{o c}(\mathrm{~V})$ & $J_{S C}\left(\mathrm{~mA} . \mathrm{cm}^{-2}\right)$ & $F F$ & $\eta(\%)$ & $\tau_{550 \mathrm{~mm}}(\%)$ \\
\hline Pt & $0.76 \pm 0.02$ & $16.2 \pm 0.2$ & $0.61 \pm 0.01$ & $7.45 \pm 0.2$ & 92.0 \\
mGOM5/Ni (water) & $0.74 \pm 0.03$ & $15.8 \pm 0.4$ & $0.64 \pm 0.02$ & $7.51 \pm 0.3$ & 80.6 \\
mGOM5/Ni (ethanol) & $0.74 \pm 0.01$ & $15.7 \pm 0.3$ & $0.64 \pm 0.01$ & $7.54 \pm 0.1$ & 91.8
\end{tabular}

[a] Jsc - short-circuit current density; Voc - open-circuit voltage; FF - fill factor; $\eta$ - power conversion efficiency; $\tau 550 \mathrm{~nm}$ - optical transmittance measured at a wavelength of 550 $\mathrm{nm}$. The standard deviation was calculated based on 3 samples and twice measured for the same cell composition and procedure.

The reason for the different optical transmittance was due to differences in the solvation effect on the graphene oxide platelets depending of the type of solvent used. This lead to films having different surface morphologies and as a result different optical transmittances. The FF value obtained for the Pt CE was relatively low when in comparison to other works due to a larger active area used $\left(0.4 \mathrm{~cm}^{2}\right)$.

The fabricated DSCs were also analyzed by electrochemical impedance spectroscopy (EIS) (Fig. 3). The correspondent EIS data was fitted according to the simplified transmission line electrical analogue model (Fig. S3). No bulk/porous diffusion should be represented due to the extremely low thickness of the graphene layer ${ }^{8}$. The mGOM5/Ni CEs exhibited a lower charge-transfer resistance (a measure of the electrocatalytic 
activity of the catalytic material at the CE/electrolyte interface20), Rct $=2.76 \Omega \mathrm{cm}^{2}$ (water) and Rct $=2.30 \Omega \mathrm{cm}^{2}$ (ethanol), compared with the resistance for the Pt CE, Rct $=5.34 \Omega \mathrm{cm}^{2}$. Consequently, the mGOM5/Ni CEs displayed a higher FF (indicative of a higher electrocatalytic activity) than for the Pt CE, despite having a slightly higher ohmic resistance (mGOM5/Ni CE, Rs = $15.1 \Omega$ (water) and Rs = 14.0 $\Omega$ (ethanol); Pt CE, Rs = 13.6 $\Omega$ ), which may hinder the flow of electrons through the cell limiting its overall efficiency. ${ }^{21}$
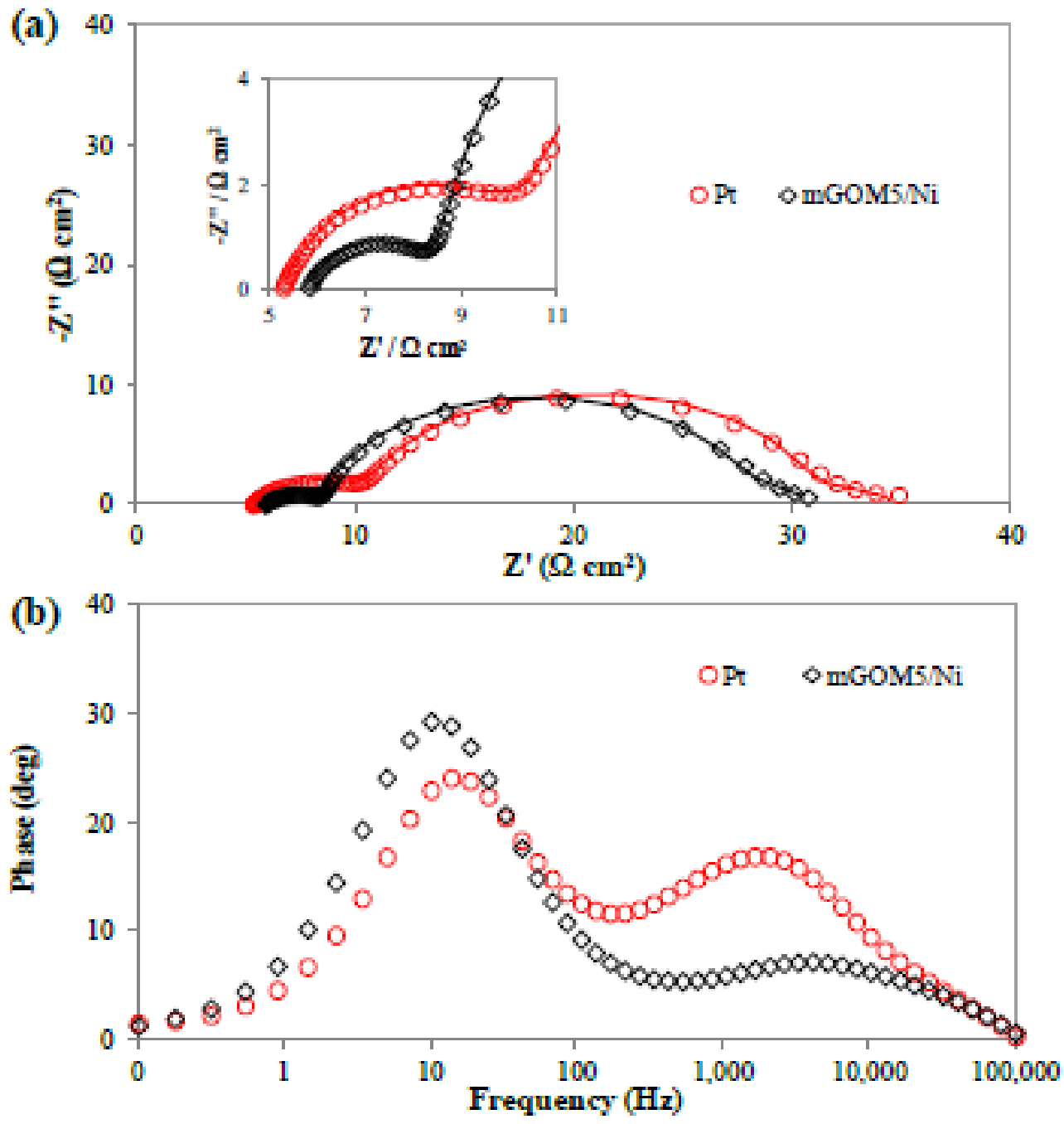

Fig. 3 Electrochemical impedance spectroscopy of DSCs assembled with mGOM5/Ni (in water) and with Pt CEs: a) Nyquist diagrams and b) Bode diagrams. Measurements carried out in the dark under a bias corresponding to the open-circuit voltage $(0.7 \mathrm{~V})$. For Nyquist diagram - Fig. 3a, full symbols represent the experimental data and solid lines represent the model fitting. ZView software (Scribner Associates Inc.) was used to process the EIS data. On the inset of the Nyquist diagrams - Fig. 3a, is represented the close-up of the semicircle corresponding to the CE's resistances. The mGOM5/Ni CEs fabricated using ethanol were not depicted due to overlap with $\mathrm{mGOM} 5 / \mathrm{Ni} \mathrm{CE}$ fabricated using water. 
The results indicate that there was a lowering of electronic resistance (catalytic and ohmic) in the CE created by the presence of Ni nanoparticles on the substrate. Therefore to better understand the role of the $\mathrm{Ni}$ nanoparticles additional DSCs with mGOM5based CEs were constructed. CEs were prepared without metal particles (mGOM5/-) and with gold nanoparticles (mGOM5/Au) (Supplementary Information section 3). These new CEs were prepared using water dispersions of mGOM5.

The mGOM5/- CE yielded a DSC with lower efficiency, having higher Rct and Rs than the mGOM5/Ni CE (Supplementary Information section 3). The DSC fabricated with the mGOM5/Au CE yielded lower efficiencies then both Pt and mGOM5/Ni CE as a direct consequence of a lower FF (higher Rct) (Supplementary Information section 3). When compared with the mGOM5/- CE the gold particles decreased the ohmic resistances between the FTO substrate and the graphene platelets $-\mathrm{Rs}=15.6 \Omega \mathrm{vs}$. Rs $=20.2 \Omega$, but did not improved their intrinsic catalytic behaviour (similar Rct, $13.5 \Omega \mathrm{cm}^{2}$ vs $12.3 \Omega$ $\mathrm{cm}^{2}$ ); such behaviour was also observed with tungsten nanoparticles. On the other hand, DSCs fabricated with CEs made of just $\mathrm{Au}$ and Ni nanoparticles yielded low efficiencies (supported by high Rct) when compared with both Pt and mGOM5/Ni CEs, despite having similar Rs (Supplementary Information section 3 ). This suggests that the $\mathrm{Ni}$ particles were not responsible for the increase of catalytic activity observed in the mGOM5/Ni CE. Furthermore, assuming that the mGOM5/- and mGOM5/Ni CEs had roughly the same thickness (because they had similar transmittances) and therefore the same available surface area for catalysis, the fact that the MGOM5 CE showed higher capacitance and higher Rct seems to indicate that the Ni nanoparticles played a role in modifying the graphene platelets such that an increase in electrocatalytic activity is observed. When graphene films are annealed in an inert atmosphere, partial restitution of the electronic conjugation takes place because of the elimination of oxygen functional groups due to thermal-induced decomposition. Additionally, graphene sheets become more ordered ${ }^{22}$ and the films smoother. The mGOM5 platelets deposited on top of the FTO/Ni became in close contact with the Ni particles during the thermal annealing. Nucleation (heteroepitaxial growth) of graphene on Ni layers can be achieved at $800{ }^{\circ} \mathrm{C}^{23}$ or above ${ }^{24}$ but these temperatures cannot be used with glass/FTO substrates. However, nickel can cause graphitization for temperatures well below, e.g. Helveg et al. ${ }^{25}$ achieved carbon nanotubes growth at $500{ }^{\circ} \mathrm{C}$ using nickel catalyst. Raman spectroscopy analysis (Table S1) revealed that the intensity ratios of the $D$ band (ID) to the $G$ band (IG), ID/IG, for $\mathrm{mGOM} 5 /-$ and $\mathrm{mGOM} 5 / \mathrm{Ni}$ films at $25^{\circ} \mathrm{C}$ (before annealing) were very similar (ca. 1.15). When they were subjected to annealing under an inert atmosphere both films suffered a reduction of ID/IG, indicating that double bonds had been partially restored due to the elimination of oxygen-containing functional groups ${ }^{22}$. Interestingly, for the electrode with the Ni particles - mGOM5/Ni, a further decrease of ID/IG is observed comparatively to the mGOM5 film (0.954 vs. 1.070, respectively). This further decrease of the ID/IG ratio seems to support the idea of extra graphene reduction by the $\mathrm{Ni}$ particles. Therefore $\mathrm{Ni}$ should have induced this partial repairing/restoration of the $\mathrm{sp}^{2}$ domains (through elimination of more oxygen functional groups or restoration of structural defects) on the mGOM5 platelets (formed by various graphene layers, (Fig. S1), making the underneath surface, as well as its connection to the FTO substrate, more electric conductive (low Rs). On the other hand, mGOM5 platelets still retained a sufficient number of catalytic sites so that the mGOM5/Ni CE could yield a high electrocatalytic activity (low Rct), given mainly by the upper surface. So the overall 
catalytic activity increased mainly, not because the number of active sites increased ${ }^{26}$, 27 , or due to increasing electrocatalysis occurring at the basal plane of the grapheme sheets ${ }^{28}$, but because the overall resistance of electronic transport was decreased. EIS analysis on half-cells ( $\mathrm{HCs}$ ), consisting of two identical FTO glass substrates coated with the catalytic materials mGOM5/Ni and $\mathrm{Pt}{ }^{20}$, yielded values of Rct and Rs consistent with the DSCs' results (Supplementary Information section 4). HCs' data was also obtained for the mGOM5/- CE, with this last having higher Rct and Rs than both Pt and mGOM5/Ni CEs. It can then be concluded that the presence of the Ni particles has a profound effect in the electrocatalytic activity of the mGOM5-based film.

\section{Conclusions}

We succeeded in developing a new transparent, low-cost and easily assembled CE that can be used in dye-sensitized solar cells that employ the $13^{-} / \mathrm{I}^{-}$redox couple. When nickel nanoparticles are placed between a FTO substrate and oxidized graphene platelets, they are capable of restoring electronic double bonds along the platelets as well as improving ohmic resistance between the catalytic material and the FTO layer. Although the ultimate efficiency of the graphene/Ni CE is approximately the same as the reference, practical and economical advantages in its use appear to exist. It is also believed that the developed structured graphene/Ni CE is compatible with other redox systems (ferrocene or cobalt) and future studies will focus on extending the use of this novel CE to these other redox electrolytes.

\section{Experimental}

Commercial graphene nanoplatelets (Grade M5, from XG Sciences) were modified (oxidized) according to the Hummer's method17. Aqueous and ethanolic dispersions of mGOM5 were prepared with ca. $0.1 \mathrm{mg}$ of catalytic material per gram of suspension.

The fabrication of the structured $\mathrm{mGOM} 5 / \mathrm{Ni}$ films involved three steps: i) electrodeposition of $\mathrm{Ni}^{2+}$ with an electrical charge of ca. $0.02 \mathrm{C}$, at a temperature of $30{ }^{\circ} \mathrm{C}$; ii) suspensions of mGOM5 were sprayed onto substrates placed on a pre-heated hot-plate at ca. $120^{\circ} \mathrm{C}$; iii) all structured films were then annealed for $15 \mathrm{~min}$ at $550{ }^{\circ} \mathrm{C}$ under an inert atmosphere and then slowly cooled $\left(<10 \stackrel{\circ}{ }\left(\bullet \mathrm{s}^{-1}\right)\right.$. mGOM5 loads of ca. 5 $-15 \mu \mathrm{g} \cdot \mathrm{cm}^{-2}$ were applied to each electrode. FTO/Au substrates for the fabrication of

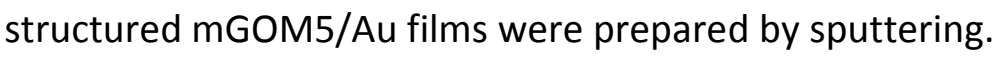

The morphology of the mGOM5 films placed on top of FTO and FTO/Ni substrates was accessed through Scanning Electron Microscopy (SEM), equipped with Energy Dispersive Spectroscopy (SEM-EDS, Hitachi SU-70 with Bruker EDS detector) and Raman imaging and spectroscopy (LabRAM HR Evolution, Horiba Scientific, excitation laser energy 2.33 $\mathrm{eV} / 532 \mathrm{~nm}$ ). The surface roughness of the films was evaluated using atomic force microscopy (AFM, Digital Instruments). Transmittance measurements of the different films were carried out using an UV/VIS/NIR spectrophotometer (Lambda 750, Perkin Elmer).

DSCs with an active area of $0.4 \mathrm{~cm} 2$ were fabricated according to the procedure described elsewhere ${ }^{16}$. N719 (1 mM in ethanol, Solaronix) was used as the dye and an iodine-based acetonitrile medium solution, as the electrolyte (EL-HPE, Dyesol). The area of catalytic material in contact with the electrolyte for $\mathrm{HCs}$ was ca. $2 \mathrm{~cm}^{2}$. 
A $150 \mathrm{~W}$ xenon light source (Oriel class A solar simulator, Newport USA) was used for obtaining the I-V curves. EIS characterization was accomplished using a ZENNIUM workstation (Ref. 2425-C, Zahner Elektrik, Germany).

\section{Acknowledgments}

This work was supported by FCT and Efacec - Engenharia e Sistemas S.A. (grant no. (SFRH/BDE/33439/2008) and projects WinDSC SI\&IDT (ref. 21539/2011), SolarConcept (ref. PTDC/EQU-EQU/120064/2010) and BI-DSC (ref. 321315) and Project Norte 070124-FEDER-000070 - Multifunctional Nanomaterials.

\section{Notes and references}

aLaboratory for Process, Environmental and Energy Engineering - Department of Chemical Engineering, Faculty of Engineering at the University of Porto, Rua Dr. Roberto Frias, s/n 4200-465 Porto Portugal, Tel: +351225081687 Fax: +351225081449 , bIFIMUP and IN-Institute of Nanoscience and Nanotechnology, Departamento de Física e Astronomia,Faculdade de Ciências, Universidade do Porto, Rua do Campo Alegre, 687, 4169-007 Porto, Portugal, ${ }^{*}$ corresponding author mendes@fe.up.pt.

**Electronic Supplementary Information (ESI) available: Details of EIS and Raman analysis. See DOI: 10.1039/c000000x/

1. B. O'Regan and M. Grätzel, Nature, 1991,353, 737-740.

2. M. Grätzel, Nature, 2001, 414, 338-344.

3. T. Kitamura, M. Maitani, M. Matsuda, Y. Wada and S. Yanagida, Chem. Lett., 2001, 30, 1054-1055.

4. T. Murakami and M. Grätzel, Inorganica Chimica Acta, 2008, 361, 572-580.

5. K. Imoto, K. Takahashi, T. Yamaguchi, T. Komura, J. Nakamura and K. Murata, Solar Energy Materials and Solar Cells, 2003, 79, 459-469.

6. A. K. Geim and K. S. Novoselov, Nat. Mater., 2007, 6, 183-191.

7. R. R. Nair, P. Blake, A. N. Grigorenko, K. S. Novoselov, T. J. Booth, T. Stauber, N. M. R. Peres and A. K. Geim, Science, 2008, 320, 1308- 1308.

8. J. D. Roy-Mayhew, D. J. Bozym, C. Punckt and I. A. Aksay, ACS Nano, 2010, 4, 6203-6211.

9. L. Tang, Y. Wang, Y. Li, H. Feng, J. Lu and J. Li, Advanced Functional Materials, 2009, 19, 2782-2789.

10. Y. Chang, S.-T. Yang, J.-H. Liu, E. Dong, Y. Wang, A. Cao, Y. Liu and H. Wang, Toxicology Letters, 2011, 200, 201-210.

11. C. Fisher, A. E. Rider, Z. Jun Han, S. Kumar, I. Levchenko and K. Ostrikov, Journal of Nanomaterials, 2012, 2012, 1-19.

12. D. W. Zhang, X. D. Li, S. Chen, H. B. Li, Z. Sun, X. J. Yin and S. M. Huang, in Nanoelectronics Conference (INEC), 2010 3rd International, ed. P. K. I. Chu, New York, 2010, pp. 610-611.

13. T. Daeneke, T.-H. Kwon, A. B. Holmes, N. W. Duffy, U. Bach and L. Spiccia, Nat Chem, 2011, 3, 211-215.

14. L. Kavan, J.-H. Yum and M. Grätzel, Nano Letters, 2011, 11, 5501-5506.

15. M. Stefik, J.-H. Yum, Y. Hu and M. Gratzel, Journal of Materials Chemistry A, 2013, 1, 4982-4987. 
16. R. Cruz, D. A. Pacheco Tanaka and A. Mendes, Solar Energy, 2012, 86, 716-724.

17. W. S. Hummers and R. E. Offeman, Journal of the American Chemical Society, 1958, 80, 1339-1339.

18. T. Ma, X. Fang, M. Akiyama, K. Inoue, H. Noma and E. Abe, Journal of Electroanalytical Chemistry, 2004, 574, 77-83.

19. K. Okada, H. Matsui, T. Kawashima, T. Ezure and N. Tanabe, Journal of Photochemistry and Photobiology A: Chemistry, 2004, 164, 193- 198.

20. A. Hauch and A. Georg, Electrochimica Acta, 2001, 46, 3457- 3466.

21. K. Aitola, J. Halme, N. Halonen, A. Kaskela, M. Toivola, A. G. Nasibulin, K. Kordás, G. Tóth, E. I. Kauppinen and P. D. Lund, Thin Solid Films, 2011, 519, 8125-8134.

22. S. J. Wang, Y. Geng, Q. Zheng and J.-K. Kim, Carbon, 2010, 48, 1815-1823.

23. H. Tanaka, S. Obata and K. Saiki, Carbon, 2013, 59, 472-478.

24. W. Choi, I. Lahiri, R. Seelaboyina and Y. S. Kang, Critical Reviews in Solid State and Materials Sciences, 2010, 35, 52-71.

25. S. Helveg, C. Lopez-Cartes, J. Sehested, P. L. Hansen, B. S. Clausen, J. R. RostrupNielsen, F. Abild-Pedersen and J. K. Norskov, Nature, 2004, 427, 426- 29.

26. L. Kavan, J. H. Yum and M. Grätzel, ACS Nano, 2010, 5, 165- 172.

27. S. Roy, R. Bajpai, A. K. Jena, P. Kumar, N. Kulshrestha and D. S. Misra, Energy Environ. Sci., 2012, 5, 7001-7006.

28. S. C. S. Lai, A. N. Patel, K. McKelvey and P. R. Unwin, Angewandte Chemie International Edition, 2012, 51, 5405-5408. 